\title{
Conclusions of the Conference
}

\author{
Captain R. Maybourn
}

As one might expect, there has ben a very considerable diversity of views as to how navigation and location on land might best be achieved or, indeed, how it is going to be achieved. However, there seems to be almost unanimous agreement that a demand for automatic vehicle location (AVL) does exist, that such facilities will be provided in the foreseable future and that the potential market is enormous.

With such a large area of common ground one might be forgiven for thinking that progress towards putting appropriate systems in place will be swift, relatively trouble-free and not too controversial. The debate over the past three days does not seem to bear this out, and some significant warnings have been signalled.

Before I remark on points of significance as they arose in specific papers, what can we identify that is not controversial? There are, I believe, a number of points on which the conference has been in agreement.

(i) The market does exist and it extends, in principle at any rate, to virtually all parts of the world.

(ii) Because of the very high cost of system development success is likely to depend upon using those which are based on proven, paid-for technology.

(iii) The user will be attracted only if the cost to him is modest or if what is offered him is demonstrably cost effective.

(iv) The direction in which technology is moving is favourable.

(v) Success will depend upon identifying what the user needs.

(vi) There is no indication as yet that the user really knows what he needs.

There are also, it seems, a few significant areas on which we are not totally agreed. In particular :

(a) is navigation or location the real objective?

(b) what accuracy is needed?

A number of warnings have been sounded, some of them amounting, perhaps, to good advice. The President, for example, in his keynote address touched on accuracy (both achievable and asked-for), the apparent mismatch between the market and the available technology and the exponential relationship between cost and accuracy, all matters echoed by other speakers during this conference.

Accuracy is as good a starting point as any. As an issue it had two quite different aspects. The first was what accuracy the user would require, and for location (particularly outside urban areas) the requirement does not seem to be unduly stringent $-100-300 \mathrm{~m}$ has been suggested. Emergency services in urban areas might, however, like something better. The second aspect of accuracy related to matters such as standards of accuracy, repeatability, absolute accuracy and so forth. Professor Ashkenazi's comments on the pitfalls in misusing coordinates were both illuminating and instructive. A conclusion I drew, with which you may not all agree, was that most accuracy problems are insignificant if the systems accuracy demanded by users is kept within sensible limits. One other warning to be highlighted related to the US Federal Radionavigation Plan, which will delineate future uses of radio navigation systems. It must be watched closely as it is revised, since it will have a major influence on terrestrial applications of radionavigation systems.

The second session of the conference dealt with civil operational requirements, and 
Pearson's paper on the need for operational research seemed to strike at the very roots of this matter. One of his final questions 'Why do I want the system? (in fact do I need it at all?)' should, perhaps, be kept clearly in mind by anyone contemplating employing $A V L$, if only to ensure that he chooses what he needs and not what someone else may think he needs.

The general case for AVL was made convincingly, albeit with qualifications by several speakers as to some of its potential applications and the perception of some potential users as to some of the benefits to be had. It was pointed out, incidentally, that potential benefits extended to areas such as the control of hazardous cargoes, monitoring taxation, gathering data for planning purposes and so forth. However, since effective control of resources is an essential element of good management any cost-beneficial method of improving control deserves serious consideration. Nevertheless, it is possible to sense scepticism amongst some potential users at least, who may wish to see AVL demonstrated by others before they are convinced of its virtues.

Technical considerations are not the only matters which concern potential users, it would seem, and both reliability of coverage (as distinct from individual equipment reliability) and acceptability to vehicle drivers were highlighted as typical of other matters not to be neglected.

The review of military operational requirements was of considerable interest, since problems were addressed which are of much greater severity than those which are met in civilian applications of AVL. None the less, cost was still an important parameter. Support of battlefield vehicles by supply vehicles seems to be a significant matter which has yet to receive adequate attention, and this might lead to systems having applications in commercial activities also.

Where unmanned vehicles are concerned, emphasis was on the integration of low-cost sensors and computer processing to produce significantly less expensive variants, rather than a single high-precision sophisticated device. We should, perhaps, as human beings take some comfort from the fact that replacing relatively inefficient but expensive people is not proving easy.

The session on terrestrial and satellite long-range systems brought us, it seemed, to the nuts and bolts of the relative merits of the several contenders for the AVL market. Surprisingly, to some of us at least, it seems Omega and Loran C, both of which seemed to have been dealt a mortal blow by satellite systems in the marine market, have a bright future in AVL and will remain operational for a long time to come.

The three papers concerned with long-range terrestrial radio systems had much in common, even though one system was based on Omega and on Loran C. Accuracies of the order of $100-300 \mathrm{~m}$ were claimed, and had inded been demonstrated, which was believed to satisfy the needs of the great majority of the market. While terrain, or the proximity of the features such as large buildings, could degrade accuracy it was agreed that these effects were not so significant as seriously to impair the usefulness of these systems. It would in any case, it was pointed out, be feasible to improve performance by incorporating DR aids (at a price) but this enhancement would not generally, it was argued, be needed by the user.

Various suggestions were offered concerning the information to be made available to the user and how it might be displayed. These ranged from simple tabulations of vehicle positions and other relevant data to colour graphics showing maps upon which vehicle positions and status were superimposed. These were not, of course, matters which were dependent upon the type of position-finding systems employed, and could be tailored to the needs of the user. None seemed to be limited in any way by current technology.

It will be recalled that before the satellite radio systems papers were presented $\mathrm{Mr}$ 
Rosetti spoke about, as he put it, 'Dreams and Reality', implying that the two might be very different. He suggested amongst other things that the availability of adequate communications links was essential for many of the AVL systems being proposed. This is a significant problem, a view which was shared by many other speakers during the conference. He also suggested that the need for navigational facilities would not be great, whereas the ability to identify locations would be very important indeed. Again this was a view receiving considerable support.

The papers which followed in this session were realistic concerning the limitations of GPS systems for AVL, especially in urban areas, and suggestions were offered for overcoming this problem by, for example, incorporating speed and direction inputs for DR back-up. Interestingly, magnetic direction sensors were regarded as entirely adequate, a position which was supported in a later paper by Mr Foster in which he described work on adaptive compasses. It is fair to say, I think, that the protagonists of GPS seem to believe that the inherent high accuracy of systems based on this technology can be exploited and that the difficulties encountered in using it over land will be successfully overcome.

The possibilities offered by displaying maps of relevant areas was discussed by Dr Pratt, who argued that map digitizing has already developed sufficiently to make this a reality within a decade. The maps for the whole of the UK, for example, could be stored on a single compact disc.

The military systems session illustrated clearly how different are military constraints and how relative are terms such as 'low cost' and 'affordable'. Clearly the need to have systems which are self-contained and independent of outside aids, which are rapid-start, which are accurate and which can tolerate rugged operating conditions is paramount, but they will never be cheap. This is a specialist area which may have some spin-off into commercial systems, but developments look mainly to be directed at different types of solution.

The session which followed considered local and urban systems. Mr Murazko, from the outset, spoke of the need for a practical, no-frill system suitable for the general motoring public in urban areas. It was refreshing to be reminded that for the UK at least, National Grid references applied to suitably indexed maps allow rapid identification of any location without any electronic intervention whatsoever.

Dr Ewen-Smith's analysis of factors affecting accuracy in a hybrid DR/signpost system was both timely and instructive. His assessment of the value of magnetometers for determining direction indicated that in spite of the unpromising magnetic environment in which they are required to operate worthwhile performance can be achieved. $\mathrm{Mr}$ Foster's paper on the adaptive compass complemented this view, and one would not disagree with his contention that such devices have a significant role to play in AVL systems.

The manner in which maps might be incorporated in position and location displays has been discussed on many occasions throughout this conference, and a number of rather different proposals have been made. The paper dealing with digital colour maps once again argued the case for digitally based maps, and this seems to be a technology which will be adapted by some at least of those developing displays.

This session ended with Mr Honey's paper. The system he was advocating has the distinction of being one of very few actually being sold in the market place. It is a simple yet practical system, and if the driver is willing to intervene to correct positional errors as the need arises - and it seems that in California at any rate he is both willing and capable - then for the urban coverage envisaged this is a solution with much to commend it. 
The final two papers discussed the use of cellular telephones for automatic vehicle tracking. I have already remarked that the availability of suitable communications links is one of the significant issues highlighted in our discussion, and cellular telephone systems may well be one answer to this problem in areas where cover is adequate. What conclusions can we draw from this conference - apart from the very real interest in AVL which has been so apparent? There are a few which seem worth mentioning.

(i) The market for AVL exists even if it has not been adequately defined. It could be both large and diverse.

(ii) The basic technology exists but a great deal of refinement may yet be necessary.

(iii) More than one solution may be appropriate depending upon whether the target is navigation or location, urban or rural and so forth.

(iv) Adequate communication links may yet, particularly in Europe perhaps, be one of the most difficult problems to resolve.

(v) Most importantly, the next decade will see widespread development and adoption of AVL systems.

\title{
'Collision Rate as a Danger Criterion for Marine Traffic'
}

\author{
from A. N. Cockcroft
}

Dr Kwik suggests (p. 203) that there are basically two ways to assess the probability of ship collision; either to estimate it directly from collision statistics or to calculate it with the aid of mathematical models of the traffic. The shortcomings of the statistical approach and the advantages of mathematical modelling are set out at length in his paper, and he concludes that the analysis of past accidents should be superseded by prospective and preventative investigation of possible accidents.

Mathematical modelling is becoming increasingly important to marine traffic safety assessment and is well established in other fields. However, it does not follow that statistical analysis is being superseded or becoming less important. Mathematical modelling must be based on the results of the relevant statistics. It would surely be preferable to consider the two approaches as complementary, rather than as distinct alternatives, one of which should be discarded.

There are also various shortcomings associated with the mathematical approach. As Dr Kwik has admitted, the human factor has always been of great importance but cannot easily be taken into account in mathematical equations. Mathematical models used to assess the probability of ship collisions are likely to be based mainly, if not entirely, on parameters which may be easily quantified including traffic density, visibility and relative velocity, as indicated in Dr Kwik's equations.

The following example, based on an analysis of sea collisions, serves to illustrate the considerable influence of human factors on the probability of ship collisions. In the Dover Strait, traffic surveys have indicated that about 300 ships pass though each day and that crossing traffic can amount to over 200 ships per day, but despite the very high frequency of broad-angle crossing encounters only two collisions between vessels crossing at a broad angle have been recorded in the last 24 years. Most of the collisions arising from broad crossing encounters have occurred in areas of low traffic density and can be attributed 